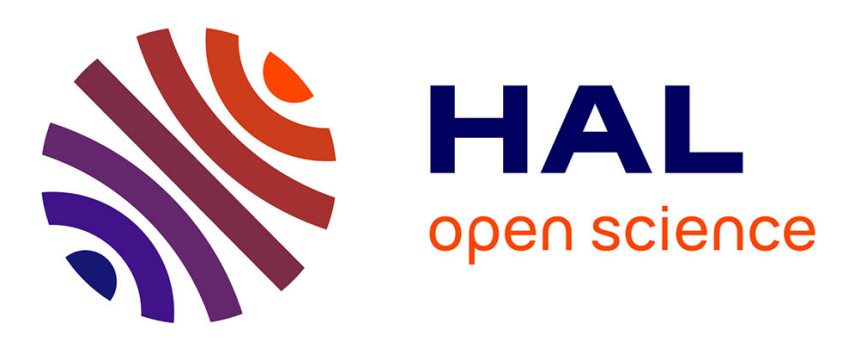

\title{
NON LINEAR HANLE EFFECT: OPTOGALVANIC DETECTION AND APPLICATION TO POWER ENHANCEMENT IN GAS LASERS
}

\author{
F. Strumia
}

\section{- To cite this version:}

F. Strumia. NON LINEAR HANLE EFFECT: OPTOGALVANIC DETECTION AND APPLICATION TO POWER ENHANCEMENT IN GAS LASERS. Journal de Physique Colloques, 1983, 44 (C7), pp.C7-117-C7-126. 10.1051/jphyscol:1983710 . jpa-00223267

\section{HAL Id: jpa-00223267 https://hal.science/jpa-00223267}

Submitted on 1 Jan 1983

HAL is a multi-disciplinary open access archive for the deposit and dissemination of scientific research documents, whether they are published or not. The documents may come from teaching and research institutions in France or abroad, or from public or private research centers.
L'archive ouverte pluridisciplinaire HAL, est destinée au dépôt et à la diffusion de documents scientifiques de niveau recherche, publiés ou non, émanant des établissements d'enseignement et de recherche français ou étrangers, des laboratoires publics ou privés. 
NON LINEAR HANLE EFFECT : OPTOGALVANIC DETECTION AND APPLICATION TO POWER ENHANCEMENT IN GAS LASERS

\author{
F. Strumia \\ Dipartimento di Fisica, P.za Tormicelli 2, 56100 Pisa, Italy
}

\begin{abstract}
Résumé - L'effet Hanle nonlinéaire est étudié à l'aide de la spectroscopie optogalvanique. L'accroissement prévu de la puissance émise des lasers à gaz est comparé aux données expérimentales.

Abstract - The Nonlinear Hanle effect was investigated by means of the optogalvanic spectroscopy. The predicted output power enhancement for gaseous lasers was compared with the available experimental results.
\end{abstract}

\title{
I - Non Linear Hanle effect
}

The Hanle effect was discovered sixty years ago/1/ and consists in a change in the polarization of the resonance radiation when an external magnetic field removes the degeneracy between the Zeeman sublevels. With the advent of the laser the Non Linear Hanle Effect (NLHE)was also discovered /2/. It manifests as an increase of the saturated absorption when the $M$ sublevels degeneracy is removed by means of a Zeeman or Stark effect.

As it is well known, in presence of a saturating laser beam of intensity $I$, the absorption coefficient of a Doppler broadened line can be written as

$$
k(\nu, S)=\frac{k_{0} /\left.\mu\right|^{2}}{\sqrt{1+S}} \exp -\left(\frac{\nu-\nu_{0}}{\Delta \nu_{D}}\right)^{2}
$$

where $R_{0} /\left.\mu\right|^{2}$ is the absorption coefficient in absence of saturation and $S=I / I$ is the saturation parameter. Expljicit expressions for I can be found in standard texbooks/3/ and depend upon the relaxation processes in the system under investigation.

In the simplest case of a $J=0 \leftrightarrow 1$ transition and a laser beam linearly polarized orthogonal to the static field or propagating along the static field ( $\triangle M=+1$ transitions) the absorption increase as a consequence of the NLHE can be easily calculated to be $/ 4,5,6 /$

$$
R(S)=\frac{k^{F}(S)}{k(S)}=\sqrt{\frac{1+2 S}{1+S}}
$$

where $R^{F}(S)$ is the saturated absorption in presence of a static field large enough to resolve the $M$ sublevels with respect to the homogeneous linewidth $\Delta \nu_{H}$, and $R(S)$ is the saturated absorption in absence of an external field. In eq. 2 it has been assumed a Doppler width much 
larger than the homogeneous 1 inewidth and a light beam of costant cross section intensity. When the laser beam is polarized for inducing only $\Delta M=0$ transitions $R^{F}(S)=R(S)$ and no absorption increase occurs as it has been experimentally demonstrated /7/. The absorption increase as expected from eq. 2 is large and independent of the frequency setting of the laser beam within the Doppler profile of the transition (fig.la). On the contrary a signal as shown in fig.lb is expected when the laser frequency is set near the line center and the absorption signal is observed as a function of the applied external field. It consists of a

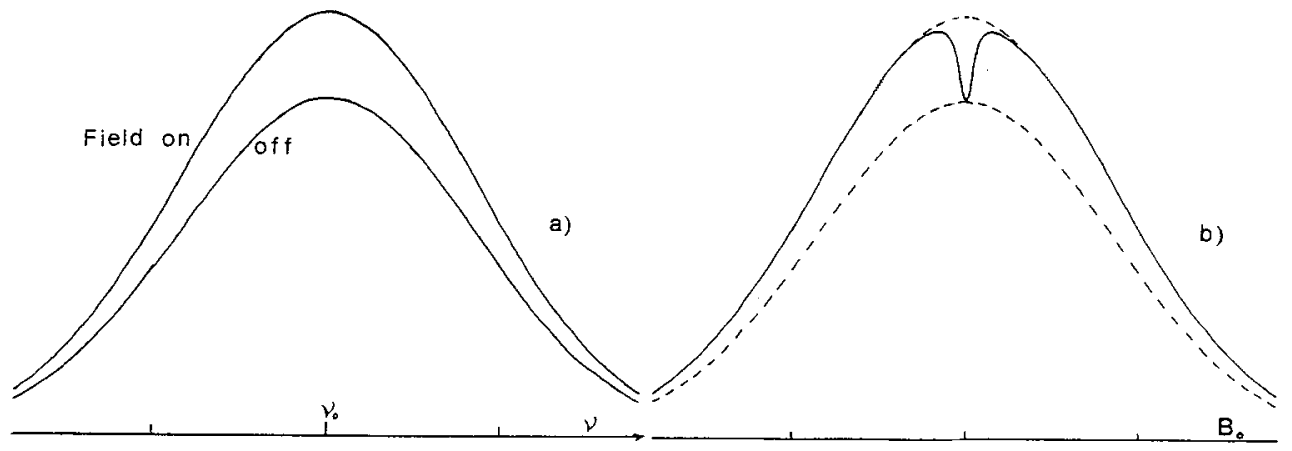

Fig. 1

difference between a Gaussian line due to the Zeeman scanning of the Doppler line by the $\Delta M= \pm 1$ transitions and a Lorentzian central dip which describes the NLHE. The HWHM of the Lorentzian is equal to the FWHM of the $\mathrm{J}=1$ level and is given by

$$
H W H M=2 g_{j} \mu_{O} B
$$

where $g_{3}$ is the Lande factor and $\mu_{0}$ the Bohr's magneton. In the more general case of a $J \leftrightarrow J$ 'transition, a density matrix treatment is necessary for describing the NLHE in both the upper and lower states of the transition /4/, however for high $\mathrm{J}$ transitions such a treatment is unpractical and cumbersome.

A simple calculation of the absorption increase in the high field limiting case ( $M$ degeneracy remove) is more convenient for understanding the magnitude of the effect. In the absence of an external field the natural quantization axis of the system is determined by the polarization and propagation direction of the laser beam. For a linearly polarized beam and a dipole interaction the quantization axis is parallel to EM field and only transitions with $\Delta M=0$ are induced. As a consequence the saturation absorption coefficient is given by

$$
k(J, S)=\sum_{-J}^{+J} k_{n}^{0}(S)=\sum_{-J}^{+J} \frac{\left|\mu_{n}^{0}\right|^{2}}{\sqrt{1+\sigma\left|\mu_{n}^{0}\right|^{2}}}
$$

where the $\left|\mu_{H}^{0}\right|^{2}$ are the dipole moments for $\Delta M=0$ transitions computed by means of the Clebsh-Gordan coefficients. This selection rule is con- 
served if a static external field is applied parallel to the quantization axis. There is no change in the saturation of the system and as a consequence no change in the absorption.

on the other hand, $\Delta M= \pm l$ transitions, are induced by a laser beam when the external static field is orthogonal to the EM field or is oriented along the beam.

In this case the $\Delta M=-1$ and $\Delta M=+1$ transitions saturate independently when the degeneracy is removed and a change in absorption is expected. It is worth noting that in the case of an inhomogeneous 1 ine whose $M$ sublevels are resolved with respect to the homogeneous width the $\Delta M=+1$ transitions starting from a given $M$ sublevel involve molecules with dif ferent velocity components along the beam direction, i.e. different molecules. Again the saturated absorption coefficient can be computed as a function of $\mathrm{J}$ and $\Delta \mathrm{J}$ selection rule by using the clebsh-Gordan coefficients

$$
k^{F}(s)=\sum_{-J}^{+J}\left(k_{H}^{+}(s)+k_{H}^{-}(s)\right)=\sum_{-J}^{+J}\left(\frac{\alpha\left|\mu_{H}^{+}\right|^{2}}{\sqrt{1+\sigma\left|\mu_{H}^{+}\right|^{2}}}+\frac{\alpha\left|\mu_{H}^{-}\right|^{2}}{\sqrt{1+\sigma\left|\mu_{H}^{-}\right|^{2}}}\right) \text { 5) }
$$

Analytical expressions for $R(S)=k^{F}(S) / R(S)$ can than be given for any $\mathrm{J}$ and $\Delta \mathrm{J}$. In the case of a plane wave such equations were reported in /6/ and /8/. In the case of a Gaussian TEM beam an analytical expres sion for. $R(S)$ can again be given for an optically thin sample as a function of $\mathrm{S}_{0}$, the saturation corresponding to the maximum intensity of the light beam $\left(I(r)=I_{0} \exp -(r / w)^{2}\right)$

$$
\begin{aligned}
& R_{Q}\left(J, S_{0}\right)=\frac{2 \sum_{-J}^{+J}\left(\sqrt{1+\frac{3}{4} S_{0} \frac{J(J+1)-M(M+1)}{J(J+1)}}-1\right)}{\sum_{-J}^{+J}\left(\sqrt{\left.1+3 S_{0} \frac{M^{2}}{J(J+1)}-1\right)}\right.} \\
& R_{R}\left(J, S_{0}\right)=R_{P}\left(J+1, S_{0}\right)=\frac{2 \sum_{-\rightarrow}^{+J}\left(\sqrt{1+\frac{3}{2} S_{0} \frac{(J+M)^{2}+3(J+M)+2}{(2 J+1)(2 J+3)}}-1\right)}{\sum_{-J}^{+J}\left(\sqrt{\left.1+6 S_{0} \frac{(J+1)^{2}-M^{2}}{(2 J+1)(2 J+3)}-1\right)}\right.}
\end{aligned}
$$

where $Q, R$ and $P$ means a $J \leftrightarrow J, J \leftrightarrow J+1$, and $J \leftrightarrow J-1$ transition respectively. In fig. 2 the computed results are shown both for integer and half integer values of $\mathrm{J}$. We found the same maximum absorption increase $(R=\sqrt{2})$ for $Q_{1}, R_{0}, R_{\frac{1}{2}}$, and $P_{1}$ transitions. By increasing the $J$ 
values, the $Q_{J}$ and $R_{J}$ lines show a rapid convergence to two limiting curves: $R_{Q}(J, S)$ increase with $J$ and $S$ up to a maximum value of 1.57 , while $R_{R}(J, S)=R_{p}(J+1, S)$ decreases to an asymptotic value of 1.27 . In both cases the effect is large and easily detectable. Only for the $\mathrm{J}=1 / 2 \leftrightarrow 1 / 2$ transition is $\mathrm{R}_{\mathrm{Q}}(1 / 2, \mathrm{~S}) \equiv \mathrm{T}$ as expected.

As for the shape of the NLHE as a function of the applied static field in the general case of a $J \leftrightarrow J$ ' transition $i t$ is worth to note that the degeneracy may be removed in the upper and lower state at a different rate depending from their indetermination and the size of the Zeeman or Stark effect. As a consequence if the Zeeman or Stark effects
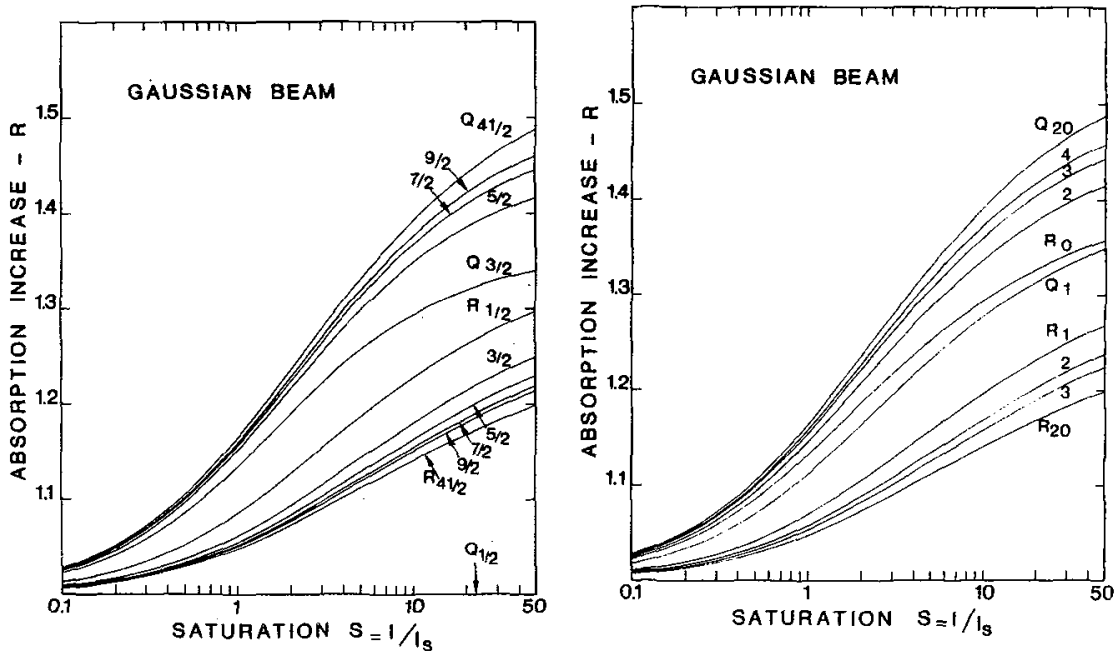

Fig. 2

are linear in the field and no HFS splitting exists within the Doppler width the NLHE is expected to consist, at the first order, of two Lorentzians with different width and amplitude. In many cases the two Lorentzians will have nearly the same width. In this case the NLHE has a Lorentzian shape.

The limiting case of high $\mathrm{J}$ transitions was investigated by using $\mathrm{CO}_{2}$ laser excitation of rotovibrational transitions of polar molecules exibiting a linear stark effect /5,7/. In fig. 3 is shown the NLHE for two transitions in $\mathrm{CH}_{3} \mathrm{OH}$ detected by means of the optoacoustic effect. As expected the NLHE is significantly smaller for the $R_{26}$ line than for the $Q_{16}$ for about the same $\mathrm{CO}_{2}$ laser power and gas pressure. A general expression for the saturation intensity Is cannot be given since it depends on the relaxation processes of any specific system 13/. In the infrared, where the rotational collisional relaxation rate is the dominant process, largely independent from $J$, Is is proportional to $p^{2}$, wherep is the gas pressure. Also this behaviour was experimentally verified /6/.

A quantitative analysis of the NLHE lineshape can be done for atomic transitions in the visible, which offer low quantum numbers. Measurements have been performed by using the optogalvanic technique in a hollow cathode discharge, which provides an easily access to the whole spectra of both volatile and refractory elements. It was demonstrated 


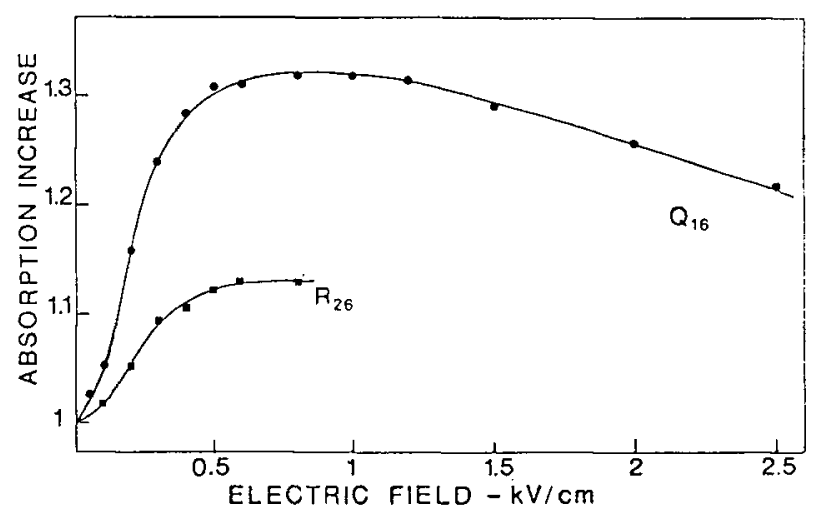

Fig. 3

that a magnetic field collinear with the laser beam and the discharge does not perturb the signals or affect the $S / N$ ratio.

Clearly resolved Zeeman pattern could be observed $/ 9 /$.

An example of the NLHE as a function of the laser frequency and for a fixed magnetic field is shown in fig. 4. The absorption increase all over the Doppler profile is clearly demonstrated.

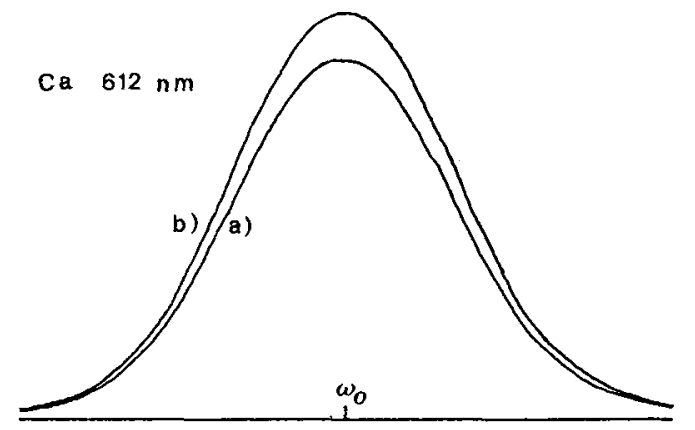

Fig. 4 - Doppler profile of the $612 \mathrm{~nm} \mathrm{Ca}$ line.

Curve a: no field, curve b: 13 Gauss.

A quantitative analysis of the shape of the NLHE as a function of the applied magnetic field will be published elsewere $/ 10 /$. In fig. 5 is shown the results for a $\mathrm{J}=0 \leftrightarrow 1$ transition in Neon at $607.4 \mathrm{~nm}$.

Since the $g_{S}$ factor of the $\mathrm{d}=1$ level is known a quantitative fit of the experimental points to the theoretical curve composed of a Lorentzian minus a Gaussian was possible /10/. The agreement was fairly good giving the following values for the independent parameters: $\Delta V_{D}=216011 \mathrm{~Hz}$; $\Delta \nu_{H}=13.7 \mathrm{MHz} ; \mathrm{R}=1.146$. The Doppler width obtained directly by scanning the line with a single mode Dye laser was found to be $2190(40) \mathrm{MHz}$. The enhancement factor $R$, as obtained from the heigth of the Lorentzian, gives the saturation parameter which is used to evaluate the contribution of the saturation to the homogeneous width. The independent determination of $S_{O}$ and $\Delta \nu_{H}$ is a unique advantage of the NLHE. 


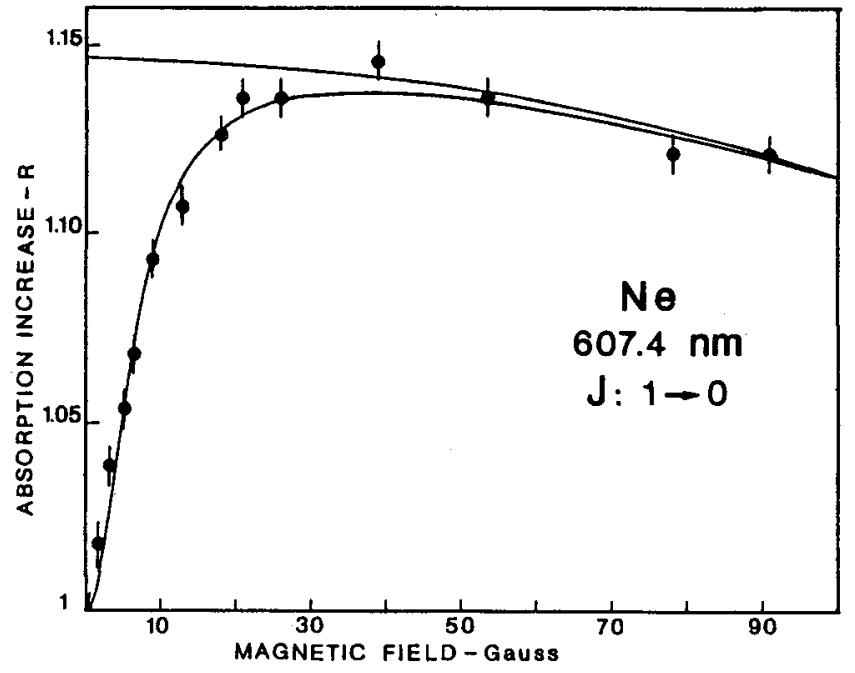

Fig. 5

The natural inewidth and the pressure broadening coefficient were then found in agreement with existing data $/ 10 /$.

For transitions with $J=1 \leftrightarrow 1$ or $J=1 \leftrightarrow 2$ a NLHE consisting of the sum of two Lorentzians could also be observed leading to an independent determination of the widths of the upper and lower levels $/ 11 /$.

\section{II - Application to the lasers output power}

In the first part we have demonstrated that the absorption coefficient of a transition is significantly increased as a consequence of the NLHE. It is obvious that the same increase is expected in the saturated gain of the laser medium when the population between the upper and Taser level is inverted.

As a consequence of the NLHE the output power of the gas laser is expected to increase when an external field is applied to the active medium. In fact such an effect was early observed in He-Ne Taser on the $0.63,1.15$, and $3.39 \mu \mathrm{m}$ 1ines $/ 12 /$. However a 11 this 1 aser 1 ines are $J=$ $=1 \leftrightarrow 2$ transitions and the relative intensity of the effect as a function of $\mathrm{J}$ as predicted by eqs. 6 and 7 cannot be tested. Moreover the output power of the laser in this case is perturbed by conpetition among the different transitions, which is also depending on the presence of an external field/T2/.

Two different cases will be considered here that would permit a better test of the predictions of the NLHE.

\section{- Optically pumped far-infrared lasers}

The far-infrared (FIR) laser emission occurs between rotational transitions of polar molecules optically pumped in an excited roto-vibrational state by means of resonant absorption of the powerful $\mathrm{CO}_{2}$ laser radiation. The pumping scheme is shown in fig.6. The pumping transition is Doppler broadened and strongly saturated while the FIR lasing 
transition is homogeneous (pressure broadening). The absence of a discharge allows an easy application of an Electric field orthogonal to the laser axis and the linear stark effect removes the degeneracy between the $M$ sublevels. Hybrid waveguides must be used that force the FIR field to be orthogonal to the static stark field. Hence only $\triangle M=+1$ selection rules are allowed for the FIR transition, while either $\Delta M=\overline{0}$ or $\Delta M=+1$ selection rules allowed for the pumping transition depending from the relative polarization of the $\mathrm{CO}_{2}$ laser beam $/ 13 /$.

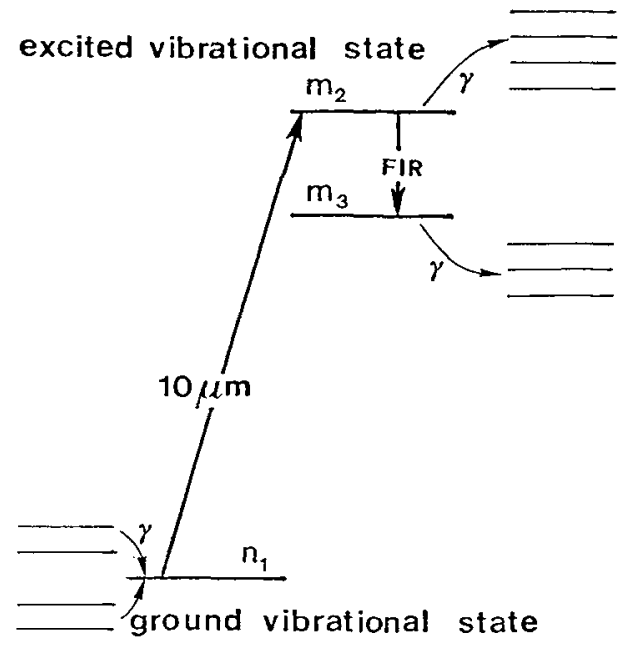

Fig. 6

For the $\Delta M= \pm l$ pump selection rules an increase in the pumping rate is expected as a consequence of the NLHE. On the contrary the stark ef fect on the FIR transition splits the line in many Stark components of which only one can be put in resonance with a FIR cavity mode. As a consequence only a gain decrease is expected on the FIR transition. In fig. 7 are reported the experimental results $/ 7 /$ obtained for FIR laser lines of about the same efficiency and wavelength.
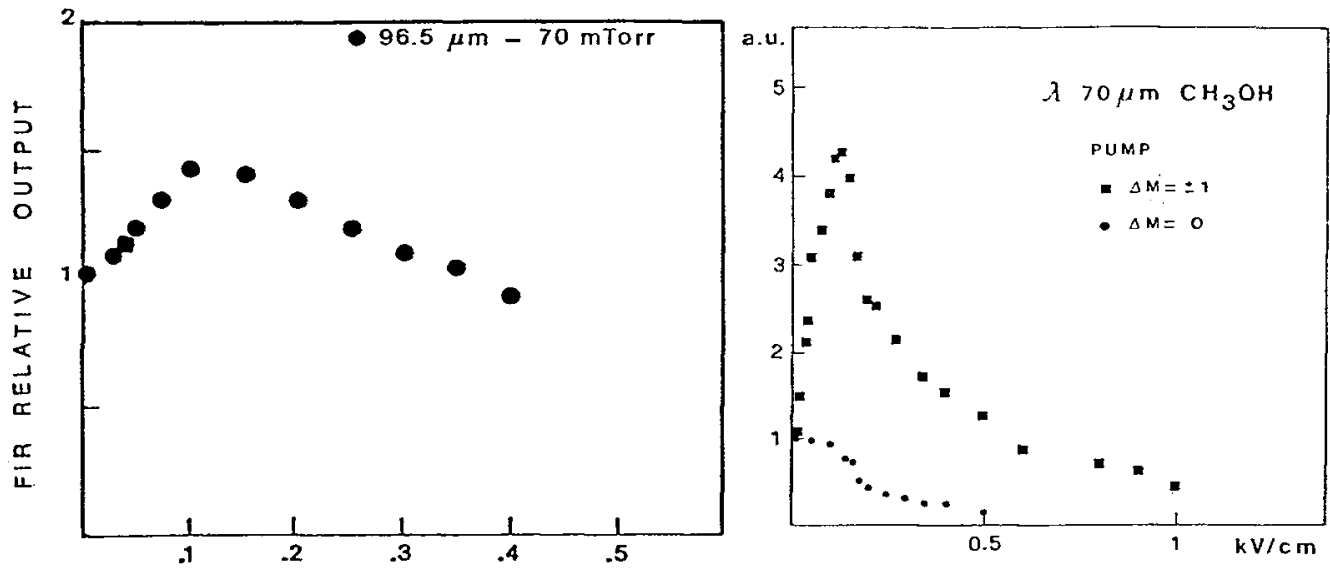

Fig. 7 
The pump transition is respectively a $Q_{9}$ line for the $70 \mu \mathrm{m}$ laser and a $R_{26}$ for the $96 \mu m$ laser. As a consequence the NLHE and the output power increase was found to be larger for the $70 \mu \mathrm{m}$ line as predicted. It is also shown that in the case of $\Delta M=0$ pump selection rule the output power showed only a power decrease, again as predicted. This result proves that the NLHE enhancement is in the pump transition since the polarization of the $70 \mu \mathrm{m}$ line is always orthogonal to the stark field.

Argon and Kripton ions laser

This lasers are the most powerful sources of $C W$ radiation in the $v i s i-$ ble and are produced in large number. All the commercial models are supplied with a solenoid that produces an axial magnetic field of about 0.1 $T$ on the discharge tube. The output power is as a consequence increased by a factor up to four.

The reason of this behaviour is not properly understood up to now.

In standard textbooks /13/ and in extensive reviews of the subject /14-16/ it is tentatively assumed that the output power enhancement may be due to an increase in the electron density in the plasma at given value of the current. However experimental investigations gave contradictory results on this points /14/. Moreover an electron density increase would produce about the same enhancement effets on all the visible laser 1 ines. On the contrary the NLHE predics a different enhancement as a func tion of $J$ and $\Delta J$ independent of the current saturation effect. In par ticular no effect is predicted for the $\mathrm{J}=1 / 2+1 / 2$ laser 1 ines. Unfortunately the available experimental data were obtained in multimode operation and only a qualitative comparison with the theory is possible. Moreover the homogeneous linewidth of the ion lasers is quite large (500 $800 \mathrm{MHz}$ ) as a consequence of the short lifetime of the lower levels and the ratio $\Delta \nu_{D} / \Delta \nu_{H}$ is about $6 \sim 7$.

In fig. 8 is shown the predicted relative gain increase $R(J, S)$ for all the CW laser lines of $\mathrm{Ar}$ and $\mathrm{Kr}$ ion lasers as a function of the saturation parameter $S_{0}$. The validity of eq. 1 has been assumed. In fact an exponent larger than $1 / 2$ may be possible as a consequence of the low $\Delta \nu_{D} / \Delta \nu_{H}$ ratio that would lead to larger $R(S)$ values.

In fig. 9 are shown experimental result obtained from the Hughes report 117/. The NLHE predicis no enhancement for the $458 \mathrm{~nm}$ laser 1 ines and the small experimental effect may be due to the expected electron density increase. A similar effect has been observed in $\mathrm{Kr}+$ red lines. The 647 $\mathrm{nm}$ line is a $\mathrm{J}=3 / 2 \leftrightarrow 5 / 2$ transition, while the $676 \mathrm{~nm}$ is $\mathrm{J}=1 / 2 \leftrightarrow \rightarrow / 2$. The first shows a large power increase as a function of the field, while the second one is practically insensible to the field. The relative enhancements of the $476 \mathrm{~nm}$ and $488 \mathrm{~nm}$ laser 1 ines is also in agreement with the prediction. The theory predicts also an equal enhancement for both the $488 \mathrm{~nm}$ and the $514 \mathrm{~nm}$ laser 1 ines. The experimental results apparently are in disagreement, the enhancement of the $514 \mathrm{~nm}$. 1 ine being larger. However the saturation intensity $I_{s}$ of this line is of about $50 \mathrm{~W} / \mathrm{cm}^{2}$ ten time larger than that of the 488 and $476 \mathrm{~nm}$ 1ines. The output power is nearly the same for the 488 and 514 ines and as a consequence the actual value of $S_{0}$ is about one order of magnitude smaller and of the order of unity for the second one. The NLHE cause a positive feedback loops: the field increases. the saturated gain which increase the laser beam intensity which increases the gain and so on. As a con- 
sequence the relative power increase is expected to be larger for low $S_{0}$ value where the slope of $R(S)$ is larger.

As for the optimum value of the magnetic field (800 roo0 Gauss), it is also in agreement with the prediction of eq. 3 if we consider the large value of the homogeneous line.

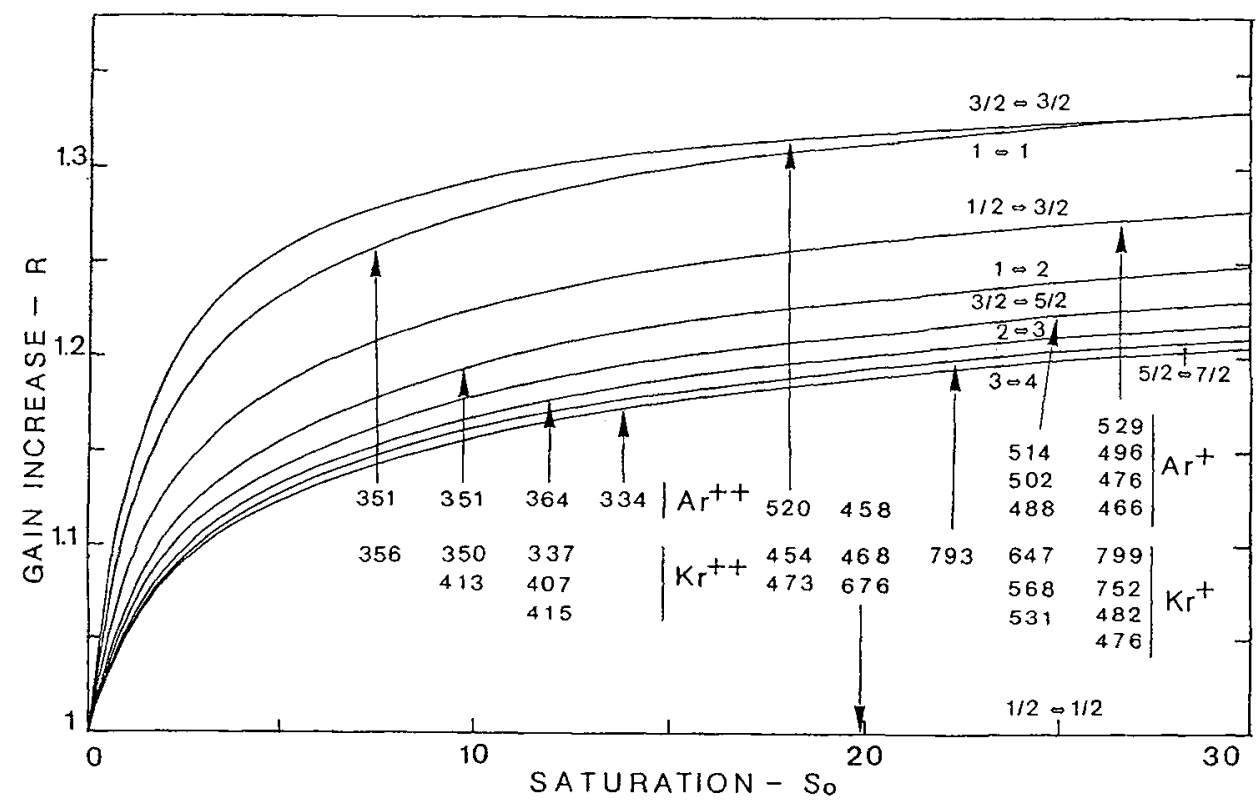

Fig. 8

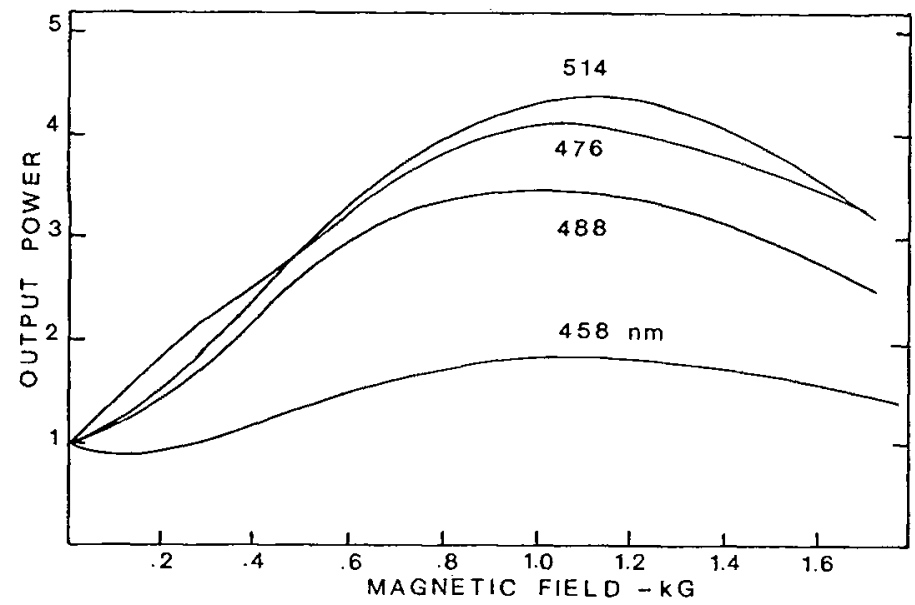

Fig. 9 
In conclusion the NLHE predictions are in qualitative agreement with the experimental results. Quantitative comparisons can be performed only with the experimental determinations of the saturated gain increase in single mode operation. At present the NLHE is however the most straight forward interpretation of the power increase in noble gases ion laser as a function of the axial magnetic field.

\section{References}

1 - W. Hanle: Naturviss. 11,691 (1923)

2 - A.C. Luntz, R.G. Brewer, K.L. Foster, J.D. Swalen: Phys .Rev.Lett. 23, 951 (1969)

3 - A. Corney: "Atomic and Laser Spectroscopy" Clarendon Press, Oxford 1977.

4 - M.S. Feld, A. Sanchez, A. Javan, B.J. Feldman: Publ. n.217 du CNRS - Paris 1974 pp. 87-104

5 - F. Strumia, M. Inguscio, A. Moretti: in "Laser Spectroscopy V" A.R.W. Mckellar, T.Oka, B.P. Stoicheff (Eds.) Springer, Berlin Heidelberg, New York 1981, pp.255

6 - M. Inguscio, A.Moretti, F.Strumia: App 1. Phys. B28, 88 (1982)

7 - M. Inguscio, A.Moretti, F.Strumia: IEEE J.Quant.Electron. QE-16, $955(1980)$

8 - F. Strumia: in "Advances in Laser Spectroscopy" F.T.Arecchi, F.Strumia, H.Walther (eds.) - Plenum, New York 1983

9 - N.Beverini, M.Galli, M.Inguscio, F.Strumia, G.Bionducci: opt. Commun. 43,261 (1982)

10 - N. Beverini, M. Inguscio, F. Strumia: Appl. Phys., in press.

11 - B. Barbieri, N.Beverini, G.Bionducci, M.Galli, M. Inguscio, F.Strumia: in "Laser Spectroscopy VI", H.P. Weber, M. Luthy(eds.) Springer, Berlin, New York, 1983

12 - M.I. Dyakonov, S.A. Fridrikhov: Soviet Phys. Uspekhi, 9, 837 (1967) and references therein.

13 - F. Strumia, M.Inguscio: in "Infrared and millimeter waves" vol. 5 K.J. Button (ed.) Academic Press, New York 1982, pp.129-213

14 - C.C. Davis, T.A. King: in "Advances in Quantum Electronics" vol.3 D.W. Goodwin (ed.) Academic Press, New York 1975, pp.169-454

15 - W.B. Bridges: in "Methods of experimental physics" vol. 15A C.L. Tang (ed.) Academic Press, New York 1979 pp.33-166

16 - M.H. Dunn, J.N. Ross: in "Progress in quantum electronics" vol.4 J.H. Sandars, S. Stenholm (eds) Pergamon Press, Oxford, New York 1977, pp.233-264

17 - W.Bridges, A.S.Ha lsted: "Gaseous I on Laser Research"Fin.Tech. Rep. Hughes Res.Lab.(1967)(unpublished)NTIS access numb.AD-814897. 